\title{
Evidence of a new metabolic capacity in an emerging diarrheal pathogen: lessons from the draft genomes of Vibrio fluvialis strains PG41 and 121563
}

Indu Khatri ${ }^{\dagger}$, Sakshi Mahajan ${ }^{\dagger}$, Chetna Dureja, Srikrishna Subramanian ${ }^{*}$ and Saumya Raychaudhuri ${ }^{*}$

\begin{abstract}
Background: Vibrio fluvialis is an emerging diarrheal pathogen for which no genome is currently available. In this work, draft genomes of two closely related clinical strains PG41 and 121563 have been explored.

Results: V. fluvialis strains PG41 and 121563 were sequenced on the Illumina HiSeq 1000 platform to obtain draft genomes of $5.3 \mathrm{Mbp}$ and $4.4 \mathrm{Mbp}$ respectively. Our genome data reveal the presence of genes involved in ethanolamine utilization, which is further experimentally confirmed by growth analysis.

Conclusions: Combined in silico and growth analysis establish a new metabolic capacity of $V$. fluvialis to harvest energy from ethanolamine.
\end{abstract}

Keywords: Metabolic fitness, Eut-operon, Vibrionaceae

\section{Background}

The genus Vibrio of the class Gammaproteobacteria is an ecologically and metabolically diverse group autochthonous to the marine, estuarine, and freshwater environment [1]. This genus comprises of nearly 100 species of which, some members are capable of causing severe diarrheal diseases, thus posing a serious threat in the developing world [2,3]. Among these, Vibrio cholerae O1/O139 and Vibrio parahaemolyticus are considered major diarrheal pathogens and are responsible for several pandemics and epidemics $[4,5]$. The other members of the Vibrionaceae family namely Vibrio mimicus and Vibirio fluvialis are also frequently found to be associated in diarrheal outbreaks [6,7].

Vibrio fluvialis is a halophilic, polarly-flagellated, Gramnegative bacterium. It was first isolated in 1975 from the stool of a diarrhea patient in Bahrain and categorized as group F Vibrio and rechristened as Vibrio fluvialis in 1981 [8]. Since its discovery, the organism has been implicated in several outbreaks and sporadic cases of

\footnotetext{
* Correspondence: krishna@imtech.res.in; saumya@rocketmail.com

tEqual contributors

CSIR-Institute of Microbial Technology, Chandigarh, India
}

diarrhea [9]. Between 1976 and 1977, 500 patients (mostly children and young adults) were reported to be infected with Vibrio fluvialis in Bangladesh with symptoms marked by vomiting, abdominal pain, moderate to severe dehydration and significant fever [10]. In the United States, Vibrio fluvialis has been associated with enterocolitis in infants [11]. In Indonesia, Vibrio fluvialis has been recognized as one of major enteric pathogen causing cholera-like diarrhea [12]. Recently, an examination of 400 non-agglutinating Vibrio species collected from patients with diarrhea in the period 2002-2009 in Kolkata, India identified 131 strains of Vibrio fluvialis of which 43 strains were suggested to be the sole pathogen and the remaining 88 strains were co-pathogens with other prominent enteric pathogens [7]. In 2009, an episode of massive diarrhea broke out in coastal regions of India following the cyclone Aila. Further investigation confirmed Vibrio fluvialis as the predominant pathogen responsible for this diarrheal outbreak [13]. Clinically, Vibrio fluvialis causes diarrhea having symptoms similar to that of cholera [14]. The organism contains El Tor-like hemoly$\sin [15]$ and exhibits cytotoxic and cell-vacuolating activity 
on HeLa cells [16]. Collectively, the information garnered from epidemiological studies clearly establishes Vibrio fluvialis as an emerging diarrheal pathogen. The situation is further aggravated by the characterization of several multi-drug resistant clinical isolates of this strain $[17,18]$.

There is now a growing realization regarding the significance of ethanolamine (EA), a small molecule present abundantly in host diet, as well as in bacterial and epithelial cells of the vertebrate intestine, that acts as an energy source for numerous bacteria including pathogens [19]. Using Salmonella enterica serovar Typhimurium as a model organism, the process of utilization of EA as an energy source has been demonstrated previously. The eut operon contains 17 genes whose concerted action converts EA into more metabolically suitable molecules. In case of Salmonella enterica, all essential proteins for EA metabolism are clustered into a multiprotein complex known as the metabolosome, which is reminiscent of the bacterial micro-compartment [20]. The presence of ethanolamine lyase (EutBC), a key enzyme of EA utilization machinery (eut) has been established in about 100 bacterial genomes [21]. In a recent effort, our group has uncovered the presence of eut operon in Vibrio alginolyticus and the capacity of this bacterium to utilize EA as a nitrogen source [22].

Taxonomically, Vibrio fluvialis belongs to the Cholerae clade. The other members of the Cholerae clade are Vibrio furnissii, Vibrio cholerae, Vibrio mimicus and Vibrio metschnikovii [23]. Genomic analysis of the members of the Cholerae clade reveals the presence of eut operon genes, thus indicating the possibility of such metabolic potential in these bacteria [22]. So far, no genome information for any strain of Vibrio fluvialis is available. This prompted us to embark on the present study to decipher the genome and examine the ability of Vibrio fluvialis to harvest energy from EA.

\section{Methods}

\section{Genome sequencing}

To pursue our interest, two Vibrio fluvialis strains namely PG41 and I21563, clinical isolates of 1998 and 2004 outbreaks respectively [5,7] were sequenced using the Illumina-HiSeq 1000 technology (See Additional file 1). For genome analysis, library preparation was carried out according to the Tru Seq DNA sample preparation protocol (Illumina, Inc., San Diego, CA) at C-CAMP, Bangalore, India. Briefly, $1 \mu \mathrm{g}$ of bacterial DNA was sheared to an average length of 300 to $400 \mathrm{bp}$, and standard blunt ending with "A" base (paired-end DNA sample preparation kit; Illumina, Inc.) was performed. Illumina index adapters were ligated to the ends of the fragments. After ligation reaction and separation of non-ligated adapters, samples were amplified by PCR for 8 cycles to selectively enrich those fragments in the library having adapter molecules at both ends. The sample was quantified and the quality was tested using a Bioanalyzer. Libraries were sequenced in a paired-end 100 base run, using TruSeq PE Cluster Kit v3-cBot-HS for cluster generation on C-bot and TruSeq SBS Kit v3-HS for sequencing on the Illumina HiSeq1000 platform according to manufacturer recommended protocols. A total of $24,420,454$ and 21,454,382 paired-end reads were obtained for $V$. fluvialis strains PG41 and I21563, respectively.

\section{Assembly and annotation}

De novo assembly approach was used to finalize the draft genomes using CLCbio wb6. The genomes were assembled with several different parameters. The genome finishing module of CLCbio was applied on the best assembly. The contigs thus obtained were scaffolded using SSPACE v2.0 scaffolder [24] and the gaps were filled by GapFiller v1.10 [25]. The gap-filled scaffolds thus obtained, were broken at the unfilled gaps. Functional annotation was carried out by RAST (Rapid Annotation using Subsystem Technology) [26], tRNA was predicted by tRNAscan-SE 1.23 [27] and rRNA genes by RNAmmer 1.2 [28].

\section{Submission of genome sequence}

This Whole Genome Shotgun project has been deposited at DDBJ/EMBL/GenBank under the accession ASXS00000000 and ASXT00000000 for Vibrio fluvialis PG41 and Vibrio fluvialis I21563 respectively. The version described in this paper is the first version ASXS01000000 and ASXT01000000.

\section{Quality assurance}

The genomic DNA was isolated from pure bacterial isolate and was further confirmed by $16 \mathrm{~S}$ rDNA gene sequencing (See Additional file 1) as well as examining certain phenotypic characteristics such as tolerance to high salt and negative for gas production in glucose-rich media which are defining characteristics of Vibrio fluvialis [16]. Multi Locus Sequence Analysis (MLSA) tree was generated with the gene sequences of six housekeeping genes fts $Z$, mreB, pyrH, recA, rpoA and topA for species characterization and confirmation. The concatenated sequences for these genes were aligned using PCMA [29]. PhyML tree was build using Topali v2.5 [30] (HKY model, 100 bootstraps) (Figure 1A). The proteins of Vibrio fluvialis strain PG41 was subjected to BLASTp at E-value $1 \mathrm{e}^{-5}$ to Vibrio fluvialis strain I21563 to find the proteome similarity percentage between the two strains.

\section{Results and discussion}

\section{Genome characteristics}

Genome of $V$. fluvialis PG41 and V. fluvialis I21563 was sequenced on Illumina HiSeq 1000 technology that resulted in a total of $24,420,454$ and $21,454,382$ paired- 


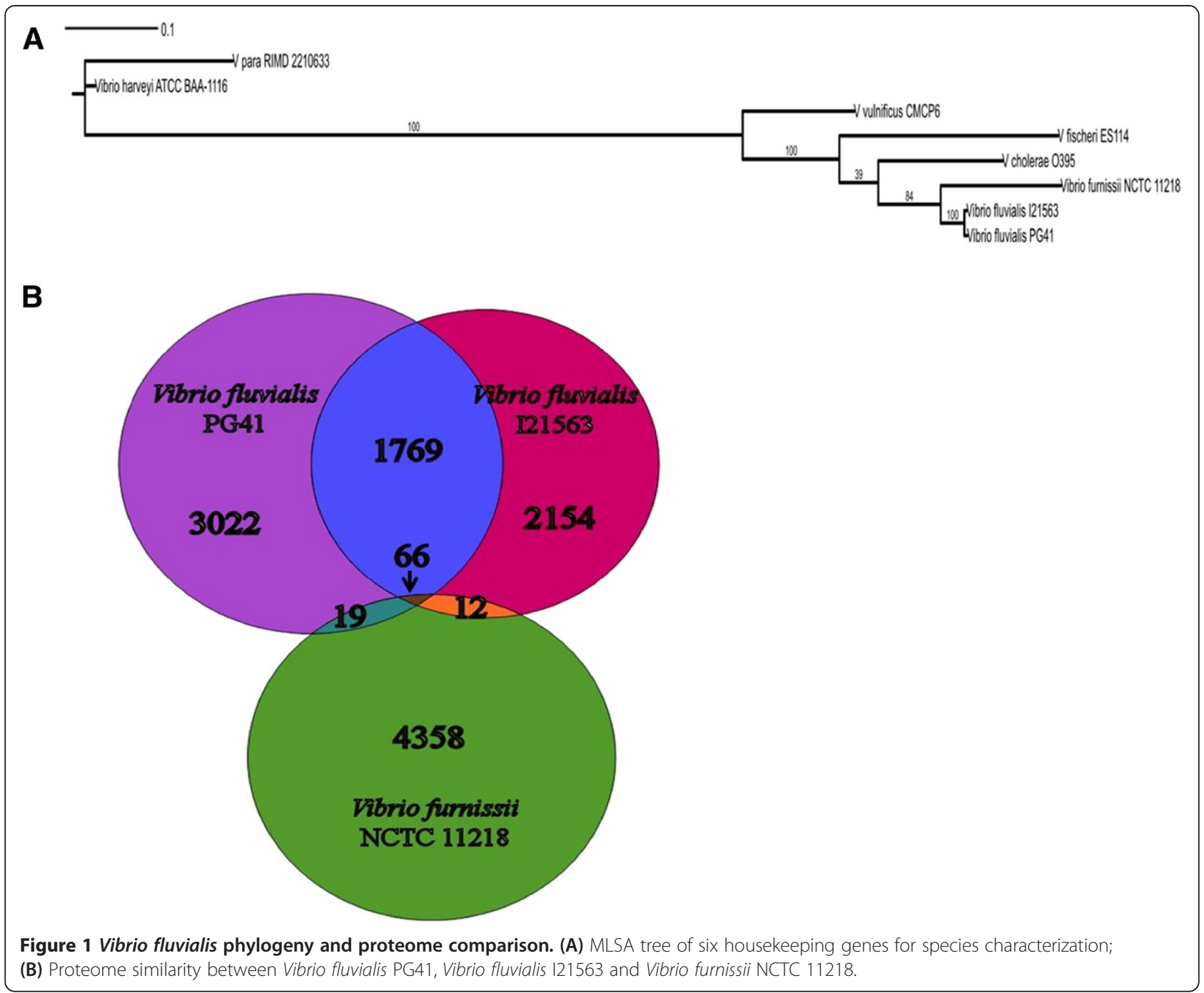

end reads of length $101 \mathrm{bp}$. A total of 24,227,857 highquality reads for $V$. fluvialis PG41 with approximately 450x coverage were assembled with CLCbio wb6 (parameters: word size 55 and bubble size 65) to obtain a draft genome of $5.3 \mathrm{Mbp}$ in 72 contigs (N50: 229,236 bp). Likewise, 21,175,217 high-quality reads from $V$. fluvialis I21563 with approximately 490× coverage were assembled with CLCbio wb6 (parameters: word size 40 and bubble size 50) to obtain a draft genome of $4.4 \mathrm{Mbp}$ in 92 contigs (N50: 104,410 bp). The genome finishing module of CLCbio followed by SSPACE v2.0 scaffolder and GapFiller v1.10 were used. The gap-filled scaffolds thus obtained, were broken at the unfilled gaps to obtain 49 contigs (N50: 236,090 bp; GC content: 48\%) of 5,343,550 bp for $V$. fluvialis PG41 and 84 contigs (N50: 109,625 bp; GC content: $50 \%$ ) of 4,371,313 bp for $V$. fluvialis I21563. The genomes contain 3 rRNA genes (5S-23S-16S) in both strains of $V$. fluvialis and 126 and 72 aminoacyl-tRNA synthetase genes in $V$. fluvialis PG41 and $V$. fluvialis
I21563, respectively. A total of 4856 coding regions were found in V. fluvialis PG41 genome, of which 3527 (72\%) could be functionally annotated whereas 3990 coding regions were identified in the genome of $V$. fluvialis strain I21563, of which 3263 (82\%) could be functionally annotated. Both strains of $V$. fluvialis, despite their differences in genome size and the total number of predicted proteins, have 1835 proteins in common at 100\% identity. Of these 1835 proteins, 66 proteins have $100 \%$ identity to the proteins of Vibrio furnissii NCTC 11218 (Figure 1B). RAST server based annotation of the whole genome, revealed the presence of 505 subsystems in Vibrio fluvialis PG41 and 493 subsystems in Vibrio fluvialis I21563 (Table 1).

\section{Existence of eut-operon}

It has been shown that EA, a small host derived molecule serves as an energy source for many bacteria including pathogens such as Salmonella enterica serotype Typhimurium and Enterohaemorrhagic Escherichia coli 
Table 1 Comparison of subsystem features between the draft genomes of Vibrio fluvialis PG41 and Vibrio fluvialis 121563

\begin{tabular}{|c|c|c|}
\hline \multirow[b]{2}{*}{ Subsystem features } & \multicolumn{2}{|c|}{ CDS present } \\
\hline & $\begin{array}{l}\text { Vibrio fluvialis } \\
\quad 121563\end{array}$ & $\begin{array}{l}\text { Vibrio fluvialis } \\
\text { PG41 }\end{array}$ \\
\hline $\begin{array}{l}\text { Cofactors, Vitamins, Prosthetic } \\
\text { Groups, Pigments }\end{array}$ & 244 & 267 \\
\hline Cell Wall and Capsule & 112 & 178 \\
\hline Virulence, Disease and Defense & 93 & 84 \\
\hline Potassium metabolism & 21 & 21 \\
\hline Miscellaneous & 33 & 33 \\
\hline $\begin{array}{l}\text { Phages, Prophages, Transposable } \\
\text { elements, Plasmids }\end{array}$ & 10 & 15 \\
\hline Membrane Transport & 164 & 180 \\
\hline Iron acquisition and metabolism & 47 & 51 \\
\hline RNA Metabolism & 149 & 113 \\
\hline Nucleosides and Nucleotides & 93 & 114 \\
\hline Protein Metabolism & 170 & 214 \\
\hline Cell Division and Cell Cycle & 30 & 28 \\
\hline Motility and Chemotaxis & 160 & 166 \\
\hline Regulation and Cell signaling & 91 & 95 \\
\hline Secondary Metabolism & 4 & 4 \\
\hline DNA Metabolism & 133 & 136 \\
\hline Regulons & 9 & 9 \\
\hline Fatty Acids, Lipids, and Isoprenoids & 114 & 120 \\
\hline Nitrogen Metabolism & 49 & 52 \\
\hline Dormancy and Sporulation & 3 & 5 \\
\hline Respiration & 127 & 129 \\
\hline Stress Response & 121 & 133 \\
\hline $\begin{array}{l}\text { Metabolism of Aromatic } \\
\text { Compounds }\end{array}$ & 17 & 17 \\
\hline Amino Acids and Derivatives & 425 & 444 \\
\hline Sulfur Metabolism & 31 & 32 \\
\hline Phosphorus Metabolism & 56 & 56 \\
\hline Carbohydrates & 427 & 494 \\
\hline
\end{tabular}

(EHEC) [19,20,31,32]. Recently, our group has established the potential of EA utilization in Vibrio alginolyticus [23]. We therefore examined the presence of genes related to EA utilization pathway in the genomes of the Vibrio fluvialis strains and compared it to homologs from Vibrio alginolyticus. It has been documented that genes from the EA utilization machinery can be clustered in the form of short or long operons [21]. The genomes of both Vibrio fluvialis strains have the short operon. Only EutBCEGPR and ethanolamine permease proteins could be identified in the draft genomes. Genes corresponding to EutRBC and ethanolamine permease are in genome context (Table 2). The percentage identity was evaluated for Eut proteins in V. alginolyticus $12 \mathrm{G} 01$ and Vibrio fluvialis (Table 1). EutBCEG of both the Vibrio fluvialis strains are $>50 \%$ identical to the respective proteins in Vibrio alginolyticus 12G01. EutPR and ethanolamine permease are 30\% identical to the proteins of Vibrio alginolyticus. We could not find any homologs of the eutD and eutQ genes in the draft genomes of Vibrio fluvialis strains PG41 and I21563. The eut operon is $100 \%$ conserved between the Vibrio fluvialis strains PG41 and I21563 and share more than 90\% sequence identity to their homologs in Vibrio furnissii NCTC 11218, the closest Vibrio for which whole genome information is available.

Vibrio fluvialis utilizes ethanolamine as an energy source As evident from the preceding section, the genome of Vibrio fluvialis strains contain genes encoding proteins of the eut operon (Table 3). To ascertain the capacity of $V$. fluvialis to utilize EA as an energy source, two clinical isolates of Vibrio fluvialis viz., PG41 and I21563 were subjected to growth analysis. The growth experiment was carried out in minimal media supplemented with EA as an energy source using a previously described procedure [22,31]. Briefly, overnight Luria broth grown cultures of these strains were further diluted and grown to bacterial $\mathrm{OD}$ of 1.0 at $37^{\circ} \mathrm{C}$ in Luria broth. The cultures were centrifuged, washed and again diluted 100-fold in M9 minimal salt medium containing

Table 2 Genomic positions of ethanolamine utilization proteins in Vibrio fluvialis PG41 and Vibrio fluvialis I21563

\begin{tabular}{|c|c|c|c|c|c|c|c|c|}
\hline \multirow[b]{2}{*}{ Eut proteins } & \multicolumn{4}{|c|}{ Vibrio fluvialis PG41 } & \multicolumn{4}{|c|}{ Vibrio fluvialis 121563} \\
\hline & Locus_tag & Contig & Start & End & Locus_tag & Contig & Start & End \\
\hline EutG & L910_2070 & 2 & 57060 & 58208 & L911_1325 & 21 & 57070 & 58218 \\
\hline EutR & L910_3169 & 3 & 301625 & 300792 & L911_0583 & 14 & 121312 & 120479 \\
\hline EutB & L910_3170 & 3 & 302044 & 303447 & L911_0584 & 14 & 121731 & 123134 \\
\hline EutC & L910_3171 & 3 & 303444 & 304286 & L911_0585 & 14 & 123131 & 123973 \\
\hline Ethanolamine permease & L910_3172 & 3 & 304317 & 305711 & L911_0586 & 14 & 124004 & 125398 \\
\hline EutE & L910_4642 & 9 & 2285 & 4987 & L911_3405 & 50 & 27410 & 24708 \\
\hline EutP & L910_1582 & 15 & 173979 & 175775 & L911_2069 & 30 & 21335 & 19671 \\
\hline
\end{tabular}


Table 3 Percentage identity of ethanolamine utilization proteins of Vibrio fluvialis strains PG41 and 121563 with Vibrio alginolyticus $12 \mathrm{G} 01$

\begin{tabular}{lcc}
\hline $\begin{array}{c}\text { Vibrio alginolyticus } \\
\text { 12G01 }\end{array}$ & $\begin{array}{c}\text { Vibrio fluvialis } \\
\text { PG41 } \\
\text { (eut operon proteins) }\end{array}$ & $\begin{array}{c}\text { Vibrio fluvialis } \\
\text { I21563 } \\
\text { (\% identity) }\end{array}$ \\
\hline EutB & 78 & 78 \\
EutC & 57 & 57 \\
EutE & 92 & 92 \\
EutG & 74 & 74 \\
EutP & 33 & 33 \\
EutR & 36 & 36 \\
eat & 37 & 37 \\
\hline
\end{tabular}

$\mathrm{KH}_{2} \mathrm{PO}_{4}\left(15 \mathrm{~g} \mathrm{l}^{-1}\right), \quad \mathrm{Na}_{2} \mathrm{PO}_{4} .7 \mathrm{H}_{2} \mathrm{O}\left(64 \mathrm{~g} \mathrm{l}^{-1}\right), \mathrm{NaCl}$ $\left(2.5 \mathrm{~g} \mathrm{l}^{-1}\right)$ supplemented with $0.1 \mathrm{mM} \mathrm{CaCl} 2,2 \mathrm{mM}$ $\mathrm{MgSO}_{4}$, and $200 \mathrm{nM}$ vitamin B12 (cyanocobalamin). To evaluate the ability of Vibrio fluvialis strains to utilize EA as a nitrogen source, $82 \mathrm{mM}$ of ethanolamine hydrochloride was added along with $0.4 \%$ glucose to minimal medium. To test the ability of these strains to use EA as a carbon source, $82 \mathrm{mM}$ of ethanolamine hydrochloride and $\mathrm{NH}_{4} \mathrm{Cl}\left(5.0 \mathrm{~g} \mathrm{l}^{-1}\right)$ were added to minimal medium. Cultures were then incubated with agitation $(200 \mathrm{rpm})$ at $37^{\circ} \mathrm{C}$ and growth was monitored over $14 \mathrm{~h}$. Our data suggests that both Vibrio fluvialis strains are capable of utilizing EA as a nitrogen source rather than a carbon source. This finding is in agreement with a similar study reported earlier for Vibrio alginolyticus [22] (Figure 2). Interestingly, Salmonella utilizes EA both as a nitrogen and carbon source [19], while EHEC and Vibrio alginolyticus prefers to use it as a nitrogen source $[22,31]$.

\section{Future directions}

Compared to other notable diarrheal pathogens of the Vibrionaceae family, our understanding of the biology of Vibrio fluvialis is not sufficiently explored. Recent works have highlighted some information on the epidemiology

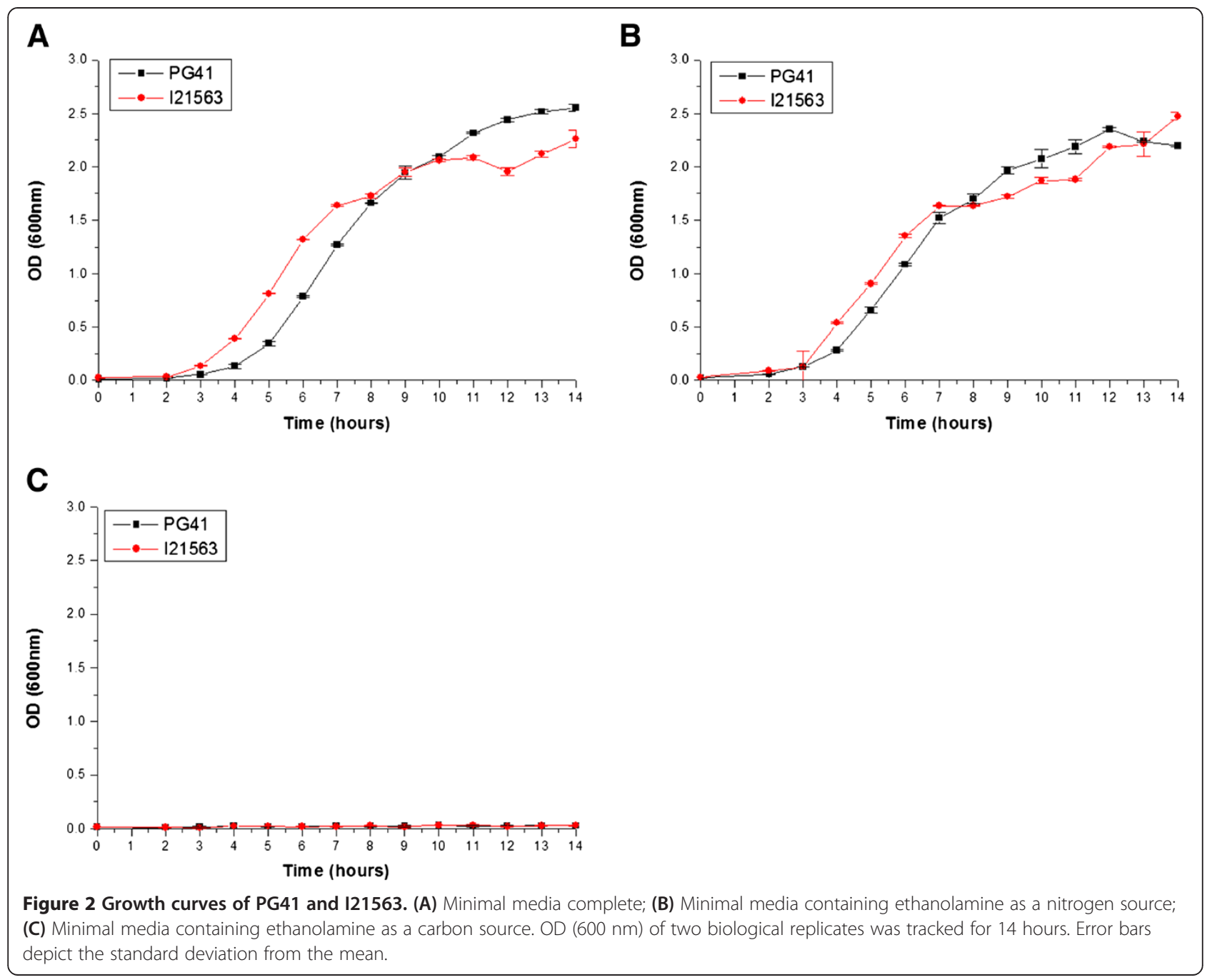


and pathogenic determinants of Vibrio fluvialis. In this regard, our draft genomes will serve as a good starting point to explore and obtain novel insights into the biology of this emerging diarrheal pathogen. Moreover, regulation of eut operon and significance of EA in controlling virulence as seen in other pathogens could be examined in Vibrio fluvialis and this is likely to shed additional light on the pathogenesis and ecology of this emerging pathogen.

\section{Availability of supporting data}

This Whole Genome Shotgun project has been deposited at DDBJ/EMBL/GenBank under the accession ASXS00000000 and ASXT00000000 for Vibrio fluvialis PG41 and Vibrio fluvialis 121563 respectively. The version described in this paper is the first version ASXS01000000 and ASXT01000000.

\section{Conclusions}

Our draft genome analysis clearly reveals the existence of the eut machinery in Vibrio fluvialis, thereby highlighting a new metabolic potential of this bacterium. Furthermore, growth analysis clearly demonstrates the capacity of this organism to harvest energy from EA preferably as a nitrogen source.

\section{Additional file}

Additional file 1: Materials and methods.

\section{Abbreviations}

PCMA: Profile Consistency Multiple Sequence Alignment; HKY: Hasegawa, Kishino and Yano; RAST: Rapid Annotation using Subsystem Technology; EA: Ethanolamine; EHEC: Enterohaemorrhagic Escherichia coli; MLSA: Multi Locus Sequence Analysis.

\section{Competing interest}

The authors declare that they have no competing interests.

\section{Authors' contributions}

SRC conceived the idea; SM and CD isolated chromosomal DNA and carried out strain identification, 165 rDNA sequencing and growth analysis of Vibrio fluvialis strains; IK and SS carried out the assembly, annotation and analysis of the genomic data. IK and SRC wrote the manuscript. All authors have read and approved the manuscript.

\footnotetext{
Acknowledgments

We gratefully acknowledge Drs. Amit Ghosh and T. Ramamurthi, National Institute of Cholera and Enteric Diseases (NICED) for generous gift of Vibrio fluvialis strains PG41 and 121563 respectively. This work was supported in part by the Department of Biotechnology (Project no. BT/PR3583/MED/30/659/ 2011-GAP 106) and CSIR Network project on Human microbiome (HUMCSIR-BSC-0119). IK and SM are supported by research fellowship from the University Grants Commission and CSIR network program on Human Microbiome (HUM-CSIR-BSC-0119) respectively. We thank the C-CAMP (http://www.ccamp.res.in/) next-generation genomics facility for help in obtaining NGS data.
}

\section{References}

1. Haley BJ, Grim CJ, Hasan NA, Choi SY, Chun J, Brettin TS, Bruce DC, Challacombe JF, Detter JC, Han CS, et al: Comparative genomic analysis reveals evidence of two novel Vibrio species closely related to $\mathrm{V}$ cholerae. BMC Microbiol 2010, 10:154.

2. Morris JG Jr: Cholera and other types of vibriosis: a story of human pandemics and oysters on the half shell. Clin Infect Dis 2003, 37:272-280.

3. Thompson FL, lida T, Swings J: Biodiversity of vibrios. Microbiol Mol Biol Rev 2004, 68:403-431. table of contents.

4. Kaper JB, Morris JG Jr, Levine MM: Cholera. Clin Microbiol Rev 1995, 8:48-86.

5. Nair GB, Ramamurthy T, Bhattacharya SK, Dutta B, Takeda Y, Sack DA: Global dissemination of Vibrio parahaemolyticus serotype O3:K6 and its serovariants. Clin Microbiol Rev 2007, 20:39-48.

6. Chitov T, Kirikaew P, Yungyune P, Ruengprapan N, Sontikun K: An incidence of large foodborne outbreak associated with Vibrio mimicus. Eur J Clin Microbiol Infect Dis 2009, 28:421-424.

7. Chowdhury G, Pazhani GP, Dutta D, Guin S, Dutta S, Ghosh S, Izumiya H, Asakura M, Yamasaki S, Takeda Y, et al: Vibrio fluvialis in patients with diarrhea, Kolkata, India. Emerg Infect Dis 2012, 18:1868-1871.

8. Lee JV, Shread P, Furniss AL, Bryant TN: Taxonomy and description of Vibrio fluvialis sp. nov. (synonym group $\mathrm{F}$ vibrios, group EF6). J Appl Bacteriol 1981, 50:73-94.

9. Igbinosa $\mathrm{EO}$, Okoh Al: Vibrio fluvialis: an unusual enteric pathogen of increasing public health concern. Int J Environ Res Public Health 2010, 7:3628-3643.

10. Huq MI, Alam AK, Brenner DJ, Morris GK: Isolation of Vibrio-like group, EF-6, from patients with diarrhea. J Clin Microbio/ 1980, 11:621-624.

11. Bellet J, Klein B, Altieri M, Ochsenschlager D: Vibrio fluvialis, an unusual pediatric enteric pathogen. Pediatr Emerg Care 1989, 5:27-28.

12. Lesmana $M$, Subekti DS, Tjaniadi $P$, Simanjuntak $\mathrm{CH}$, Punjabi $\mathrm{NH}$ Campbell JR, Oyofo BA: Spectrum of vibrio species associated with acute diarrhea in North Jakarta, Indonesia. Diagn Microbiol Infect Dis 2002, 43:91-97

13. Bhattacharjee S, Bhattacharjee S, Bal B, Pal R, Niyogi SK, Sarkar K: Is Vibrio fluvialis emerging as a pathogen with epidemic potential in coastal region of eastern India following cyclone Aila? J Health Popul Nutr 2010, 28:311-317.

14. Allton DR, Forgione MA Jr, Gros SP: Cholera-like presentation in Vibrio fluvialis enteritis. South Med J 2006, 99:765-767.

15. Kothary $\mathrm{MH}$, Lowman $\mathrm{H}, \mathrm{McC}$ cardell BA, Tall BD: Purification and characterization of enterotoxigenic El Tor-like hemolysin produced by Vibrio fluvialis. Infect Immun 2003, 71:3213-3220.

16. Chakraborty R, Chakraborty S, De K, Sinha S, Mukhopadhyay AK, Khanam J, Ramamurthy T, Takeda Y, Bhattacharya SK, Nair GB: Cytotoxic and cell vacuolating activity of Vibrio fluvialis isolated from paediatric patients with diarrhoea. J Med Microbiol 2005, 54:707-716.

17. Ahmed AM, Shinoda S, Shimamoto T: A variant type of Vibrio cholerae SXT element in a multidrug-resistant strain of Vibrio fluvialis. FEMS Microbiol Lett 2005, 242:241-247.

18. Chowdhury G, Pazhani GP, Nair GB, Ghosh A, Ramamurthy T: Transferable plasmid-mediated quinolone resistance in association with extendedspectrum beta-lactamases and fluoroquinolone-acetylating aminoglycoside-6'-N-acetyltransferase in clinical isolates of Vibrio fluvialis. Int J Antimicrob Agents 2011, 38:169-173.

19. Garsin DA: Ethanolamine utilization in bacterial pathogens: roles and regulation. Nat Rev Microbiol 2010, 8:290-295.

20. Kofoid E, Rappleye C, Stojiljkovic I, Roth J: The 17-gene ethanolamine (eut) operon of Salmonella typhimurium encodes five homologues of carboxysome shell proteins. J Bacteriol 1999, 181:5317-5329.

21. Tsoy $O$, Ravcheev D, Mushegian A: Comparative genomics of ethanolamine utilization. J Bacterio/ 2009, 191:7157-7164.

22. Khatri N, Khatri I, Subramanian S, Raychaudhuri S: Ethanolamine utilization in Vibrio alginolyticus. Biol Direct 2012, 7:45.

23. Sawabe T, Kita-Tsukamoto K, Thompson FL: Inferring the evolutionary history of vibrios by means of multilocus sequence analysis. J Bacterio/ 2007, 189:7932-7936.

24. Boetzer M, Henkel CV, Jansen HJ, Butler D, Pirovano W: Scaffolding preassembled contigs using SSPACE. Bioinformatics 2011, 27:578-579.

25. Nadalin F, Vezzi F, Policriti A: GapFiller: a de novo assembly approach to fill the gap within paired reads. BMC Bioinforma 2012,

13 Suppl 14:S8. 
26. Aziz RK, Bartels D, Best AA, Dejongh M, Disz T, Edwards RA, Formsma K, Gerdes S, Glass EM, Kubal M, et al: The RAST Server: rapid annotations using subsystems technology. BMC Genomics 2008, 9:75.

27. Lowe TM, Eddy SR: tRNAscan-SE: a program for improved detection of transfer RNA genes in genomic sequence. Nucleic Acids Res 1997, 25:955-964.

28. Lagesen $\mathrm{K}$, Hallin P, Rodland EA, Staerfeldt HH, Rognes T, Ussery DW: RNAmmer: consistent and rapid annotation of ribosomal RNA genes. Nucleic Acids Res 2007, 35:3100-3108.

29. Pei J, Sadreyev R, Grishin NV: PCMA: fast and accurate multiple sequence alignment based on profile consistency. Bioinformatics 2003, 19:427-428.

30. Milne I, Lindner D, Bayer M, Husmeier D, McGuire G, Marshall DF, Wright F: TOPALi v2: a rich graphical interface for evolutionary analyses of multiple alignments on HPC clusters and multi-core desktops. Bioinformatics 2009, 25:126-127.

31. Bertin Y, Girardeau JP, Chaucheyras-Durand F, Lyan B, Pujos-Guillot E, Harel J, Martin C: Enterohaemorrhagic Escherichia coli gains a competitive advantage by using ethanolamine as a nitrogen source in the bovine intestinal content. Environ Microbiol 2011, 13:365-377.

32. Kendall MM, Gruber CC, Parker $C T$, Sperandio V: Ethanolamine controls expression of genes encoding components involved in interkingdom signaling and virulence in enterohemorrhagic Escherichia coli 0157:H7. MBio 2012, 3.

doi:10.1186/1757-4749-5-20

Cite this article as: Khatri et al:: Evidence of a new metabolic capacity in an emerging diarrheal pathogen: lessons from the draft genomes of Vibrio fluvialis strains PG41 and 121563. Gut Pathogens 2013 5:20.

\section{Submit your next manuscript to BioMed Central and take full advantage of:}

- Convenient online submission

- Thorough peer review

- No space constraints or color figure charges

- Immediate publication on acceptance

- Inclusion in PubMed, CAS, Scopus and Google Scholar

- Research which is freely available for redistribution 